\title{
Exchange Rate Returns Standardized by Realized Volatility are (Nearly) Gaussian*
}

by

\author{
Torben G. Andersen ${ }^{\mathrm{a}}$, Tim Bollerslev ${ }^{\mathrm{b}}$, Francis X. Diebold ${ }^{\mathrm{c}}$ and Paul Labys ${ }^{\mathrm{d}}$
}

September 1999

This version: October 26, 1999

\footnotetext{
* This work was supported by the National Science Foundation. We are grateful to Olsen and Associates for making available the intraday exchange rate quotations. For useful comments we thank David Backus, Rob Engle, John Geweke, and seminar participants at New York University.

${ }^{a}$ Department of Finance, Kellogg Graduate School of Management, Northwestern University, phone: 847-467-1285, e-mail: t-andersen@nwu.edu

${ }^{\mathrm{b}}$ Department of Economics, Duke University, and NBER, phone: 919-660-1846, e-mail: boller@econ.duke.edu

${ }^{c}$ Department of Finance, Stern School of Business, New York University, and Departments of Economics and Statistics, University of Pennsylvania, and NBER, phone: 610-585-4057, e-mail: fdiebold@stern.nyu.edu

${ }^{\mathrm{d}}$ Graduate Group in Economics, University of Pennsylvania, 3718 Locust Walk, Philadelphia, PA 19104-6297, phone: 215-545-0450, e-mail: labys@ssc.sas.upenn.edu
}

Copyright (C) 1999 T.G. Andersen, T. Bollerslev, F.X. Diebold, and P. Labys 


\section{Introduction and Basic Ideas}

The prescriptions of modern financial risk management hinge critically on the associated characterization of the distribution of future returns (cf., Diebold, Gunther and Tay, 1998, and Diebold, Hahn and Tay, 1999). Because volatility persistence renders high-frequency returns temporally dependent (e.g., Bollerslev, Chou and Kroner, 1992), it is the conditional return distribution, and not the unconditional distribution, that is of relevance for risk management. This is especially true in high-frequency situations, such as monitoring and managing the risk associated with the day-to-day operations of a trading desk, where volatility clustering is omnipresent.

Exchange rate returns are well-known to be unconditionally symmetric but highly leptokurtic. Standardized daily or weekly returns from ARCH and related stochastic volatility models also appear symmetric but leptokurtic; that is, the distributions are not only unconditionally, but also conditionally leptokurtic, although less so than unconditionally. ${ }^{1}$ A sizable literature explicitly attempts to model the fat-tailed conditional distributions, including, for example, Bollerslev (1987), Engle and Gonzalez-Rivera (1991), and Hansen (1994).

Let us make the discussion more precise. Assuming that return dynamics operate only through the conditional variance, a standard decomposition of the time-t return (innovation) is

$$
r_{t}=\sigma_{t} \varepsilon_{t}
$$

iid

where $\sigma_{t}$ refers to the time-t conditional standard deviation, and $\varepsilon_{t} \sim(0,1)$. Thus, given $\sigma_{t}$ it would be straightforward to back out $\varepsilon_{t}$ and assess its distributional properties. Of course, $\sigma_{t}$ is

${ }^{1}$ As argued by Hsieh (1989), a higher conditional than unconditional kurtosis may be interpreted as evidence of model misspecification. 
not directly observable. When using an estimate of $\sigma_{t}$ the distributions of the resulting standardized returns are typically found to be fat-tailed, or leptokurtic. ${ }^{2}$

The main focus of the present paper is similar. However, there is an important distinction: our volatility measure is fundamentally different from the ARCH and related estimators that have featured prominently in the literature, and hence our estimates of the conditional distribution differ as well. In particular, we rely on so-called realized volatility measures constructed from highfrequency intraday returns, as previously analyzed by Schwert (1990), Hsieh (1991), Andersen and Bollerslev (1998), and Andersen, Bollerslev, Diebold and Labys (1999a), among others. Importantly, in this situation, the assumption of an underlying continuous-time diffusion process implies that the distribution of $\varepsilon_{t}$ should be Gaussian. This contrasts with the conditional distributions of discrete time parametric GARCH and stochastic volatility models, for which theory makes no particular prediction regarding the distribution of the standardized returns. Based on ten years of high-frequency returns for the Deutschemark - U.S. Dollar (DM/\$) and Japanese Yen - U.S. Dollar (Yen/\$) exchange rates, we will show that the actual empirical distributions are in fact consistent with this theoretical prediction.

We proceed as follows. In order to establish a proper benchmark, section 2 provides a characterization of the distribution of the daily unstandardized returns. In section 3 we characterize the distribution of the daily returns when standardized by our realized univariate volatility measures, and in section 4 we characterize the distribution of the returns when standardized by the realized volatilities in a multivariate fashion. For comparison, in section 5 we

\footnotetext{
2 This result has motivated the practical use of various "fudge-factors" relative to the standard normal quantiles in the construction of Value-at-Risk type statistics.
} 
examine the distribution of returns standardized by $\operatorname{GARCH}(1,1)$ volatilities, along with the distribution of returns standardized by one-day-ahead volatility forecasts from a simple $\operatorname{ARMA}(1,1)$ model fit directly to realized volatility. We conclude in section 6.

\section{Unstandardized Returns}

Our empirical analysis is based on a 10 -year time series of 5-minute DM/\$ and $\mathrm{Yen} / \$$ returns from December 1, 1986 through December 1, 1996. The data were kindly supplied by Olsen \& Associates. After omitting weekend and other holiday non-trading periods, as detailed in Andersen, Bollerslev, Diebold, and Labys (1999a), we are left with a total of T=2,445 complete days, each of which consists of 2885 -minute returns. From these we proceed to construct time series of continuously compounded 30-minute and daily returns.

We begin our analysis with a summary of the distributions for the unstandardized, or raw, daily DM $/ \$$ and Yen $/ \$$ returns. The results appear in Table 1 and Figures 1 through 3. Consistent with the extant literature, the s-shaped quantile-quantile plots for the two marginal distributions in the top panel of Figure 1 indicate that both returns are symmetric but fat-tailed relative to the normal distribution. The statistics reported in the first panel of Table 1 confirm that impression: the sample skewness is near 0.0 for both series, but the sample kurtoses are well above the normal value of 3.0.

Turning to the joint distribution, not surprisingly, the two rates show considerable dependence, with a sample correlation of 0.66. This high degree of dependence is further underscored by the bivariate scatterplot in the top panel of Figure 2, which also clearly illustrates the marginal fat tails in terms of the many outliers relative to the tight ellipsoid expected under bivariate normality. 
Finally, we consider the conditional distribution of the unstandardized returns, as summarized by the autocorrelations for each of the two daily squared return series and the cross product of the two rates. The relevant correlograms to a displacement of 100 days, along with the conservative Bartlett standard errors, appear in the top panel of Figure 3. Again, directly in line with existing evidence in the literature, the results indicate highly persistent conditional variance and covariance dynamics.

\section{Univariate Standardization by Realized Volatility}

In the absence of any short-run predictability in the mean, which is a good approximation for the two exchange rates analyzed here, the univariate return series are naturally decomposed as iid

$r_{t}=\sigma_{t} \varepsilon_{t}$, where $\varepsilon_{t} \sim(0,1)$, and $\sigma_{t}$ is the time-t conditional standard deviation. On rearranging this decomposition, we obtain the $\sigma$-standardized return,

$$
\varepsilon_{t}=\frac{r_{t}}{\sigma_{t}}
$$

on whose distribution and dependence structure we now focus.

In practice, of course, $\sigma_{t}$ is unknown and must be estimated. ${ }^{3}$ Many volatility models have been proposed in the literature. However, as formally shown by Andersen, Bollerslev, Diebold and Labys (1999a), in a continuous time setting the ex-post volatility over a day may be estimated to any desired degree of accuracy by summing sufficiently high-frequency returns within the day. Following this analysis we shall refer to the corresponding measures as realized volatilities.

In order to define formally our daily realized volatilities, let the two series of 30-minute

\footnotetext{
${ }^{3}$ In an abuse of notation, we will continue to use $\sigma_{t}$ to denote an estimate of the volatility, as the meaning will be clear from context.
} 
$\mathrm{DM} / \$$ and Yen/\$ returns be denoted by $\Delta \log D_{(48), t}$ and $\Delta \log Y_{(48), t}$, respectively, where $t=1 / 48$, $2 / 48, \ldots, 2,445$, and " 48 " refers to the 48 30-minute intervals in the 24 -hour trading day. From these $48 \cdot 2,445=117,36030$-minute returns, we estimate the daily variances by simply summing the 48 squared returns within each day. That is,

$$
\begin{gathered}
\sigma_{D t}^{2}(R V) \equiv \Sigma_{j=1, . ., 48}\left(\Delta \log D_{(48), t-1+j / 48}\right)^{2} \\
\sigma_{Y t}^{2}(R V) \equiv \Sigma_{j=1, . ., 48}\left(\Delta \log Y_{(48), t-1+j / 48}\right)^{2},
\end{gathered}
$$

where $t=1,2, \ldots, 2,445$, and "RV" stands for "realized volatility." Our choice of half-hour returns is motivated by the analysis in Andersen, Bollerslev, Diebold and Labys (1999b), which suggests that in the present context 30-minute sampling provides a reasonable balance between the salient market microstructure frictions at the very highest sampling frequencies on the one hand, and the accuracy of the continuous record asymptotics underlying the estimators on the other.

We now proceed to examine the $\sigma(R V)$-standardized returns for each of the two currency series. The quantile-quantile plots in the middle panel of Figure 1 look radically different from those in the top panel. In particular, they are now nearly linear, indicating that a Gaussian distribution affords a close approximation to each of the marginal distributions. The diagnostic statistics in the second panel of Table 1 confirm that impression: the distributions of the $\sigma(R V)$ standardized daily returns are remarkably close to a standard normal. The means are near zero, the standard deviations are close to one, the skewnesses coefficients are close to zero, and the 
coefficients of kurtosis are near three. ${ }^{4}$ If anything, the distributions appear slightly thin-tailed, or platykurtic. Interpreting the realized volatility as an ideal measure of the rate of information flow to the market, these findings are therefore consistent with the distributional assumptions underlying the Mixture-of-Distributions-Hypothesis (MDH) as originally advocated by Clark (1973); see also Tauchen and Pitts (1983) and Taylor (1986).

Proceeding to the joint unconditional distribution of the $\sigma(R V)$-standardized returns, not surprisingly, we see from the second panels of Table 1 and Figure 2 that the correlation remains high. Interestingly, however, the outliers in the joint density have been largely eliminated. As for the conditional distribution, the correlograms for the squares and the cross product of the daily $\sigma(R V)$-standardized returns indicate the absence of any remaining conditional variance dynamics for the $\mathrm{DM} / \$$ rate, and a great reduction in the conditional variance dynamics for the $\mathrm{Yen} / \$$ rate. Meanwhile, the autocorrelations for the cross product of the standardized returns decay more slowly than the autocorrelations for the product of the raw returns. Thus, although the univariate standardization has largely eliminated the conditional variance dynamics, it has actually magnified the conditional covariance dynamics. Elimination of both requires a multivariate standardization, to which we now turn.

\footnotetext{
${ }^{4}$ In an independent study, Bollen and Inder (1999) have recently observed that the distribution of $\sigma(R V)$-standardized daily S\&P500 futures returns also appear approximately Gaussian.
} 


\section{Multivariate Standardization by Realized Volatility}

With a slight abuse of notation, the multivariate case is conveniently written as,

$$
r_{t}=P_{t} \varepsilon_{t}
$$

iid

where both $r_{t}$ and $\varepsilon_{t} \sim(0,1)$ are now $\mathrm{Nx} 1$ vectors, and $\mathrm{P}_{\mathrm{t}}$ refers to the $\mathrm{NxN}$ matrix square-root of the time-t conditional covariance matrix for the raw returns, $\Sigma_{t}$, so that in particular $P_{t} P_{t}^{\prime}=\Sigma_{t}$.

Of course, the matrix square-root operator is not unique. For concreteness, we rely here on the unique NxN lower-triangular Cholesky factorization. The corresponding P-standardized return vector is then readily defined as,

$$
\varepsilon_{t}=P_{t}^{-1} r_{t}
$$

which, in general, will differ from the corresponding vector of stacked univariate $\sigma$-standardized returns. In particular, we have

$$
\Sigma_{t}=\left[\begin{array}{cc}
\sigma_{D D t}^{2} & \sigma_{D Y t} \\
\sigma_{D Y t} & \sigma_{Y Y t}^{2}
\end{array}\right]=P_{t} P_{t}^{\prime}=\left[\begin{array}{cc}
p_{11 t} & 0 \\
p_{21 t} & p_{22 t}
\end{array}\right]\left[\begin{array}{cc}
p_{11 t} & p_{21 t} \\
0 & p_{22 t}
\end{array}\right]=\left[\begin{array}{cc}
p_{11 t}^{2} & p_{21} p_{11 t} \\
p_{11 t} p_{21 t} & p_{21 t}^{2}+p_{22 t}^{2}
\end{array}\right]
$$

where we have arbitrarily arranged the bivariate returns as $(\mathrm{DM} / \$, \mathrm{Yen} / \$)$. Upon matching terms, it follows that 


$$
\begin{aligned}
& p_{11 t}=\sigma_{D D t} \\
& p_{21 t}=\frac{\sigma_{D Y t}}{\sigma_{D D t}} \\
& p_{22 t}=\sqrt{\sigma_{Y Y t}^{2}-\frac{\sigma_{D Y t}^{2}}{\sigma_{D D t}^{2}}},
\end{aligned}
$$

so,

$$
P_{t}^{-1}=\frac{1}{p_{11 t} p_{22 t}}\left[\begin{array}{cc}
p_{22 t} & 0 \\
-p_{21 t} & p_{11 t}
\end{array}\right]=\left[\begin{array}{cc}
\frac{1}{p_{11 t}} & 0 \\
\frac{-p_{21 t}}{p_{11 t} p_{22 t}} & \frac{1}{p_{22 t}}
\end{array}\right]=\left[\begin{array}{cc}
\frac{1}{\sigma_{D D t}} & 0 \\
-\frac{\sigma_{D Y t}}{\sigma_{D D t}^{2}} \cdot \frac{1}{\sqrt{\sigma_{Y Y t}^{2}-\sigma_{D Y t}^{2} / \sigma_{D D t}^{2}}} & \frac{1}{\sqrt{\sigma_{Y Y t}^{2}-\sigma_{D Y t}^{2} / \sigma_{D D t}^{2}}}
\end{array}\right] .
$$

Hence, P-standardization of a time-t return vector is equivalent to element-by-element, or univariate, $\sigma$-standardization only in the special and counterfactual case of $\sigma_{D Y t}=0$. Moreover, the ordering matters. The P-standardization simply $\sigma$-standardizes the return placed first in the ordering, whereas it substitutes a linear combination of the two unstandardized returns for the second return. ${ }^{5}$

Of course, the $P_{t}$ matrix involves both exchange rate variances and their covariance. Analogous to our realized variance estimator, the realized covariance is readily defined as

\footnotetext{
${ }^{5}$ This mirrors the dependence on the ordering of the variables in the analysis of Vector AutoRegressions (VARs) as identified by a Wold Causal Chain.
} 
the sum of the intra-day cross products:

$$
\sigma_{D Y}(R V) \equiv \Sigma_{j=1, \ldots, 48} \Delta \log D_{(48), t-1+j / 48} \cdot \Delta \log Y_{(48), t-1+j / 48} .
$$

Armed with these realized variances and covariances, we now proceed to construct and examine $P(R V)$-standardized returns.

The results are reported in the third panel of Table 1 and Figures 1-3. The differences, as

expected, arise primarily in the multivariate dimensions of the distribution. The sample correlation between the bivariate $P(R V)$-standardized returns, as reported in Table 1 , is greatly reduced from 0.66 to only 0.08 . Moreover, the scatterplot reported in the third panel of Figure 2 now appears spherical, confirming the negligible correlation. Importantly, the correlogram for the cross products of the daily $P(R V)$-standardized returns, reported in the third panel of Figure 3 , confirms that the conditional covariance dynamics have been eliminated. The differences, however, are not exclusively in terms of the multivariate features. In particular, the $P(R V)$ standardization also produces an improved correlogram for the Yen $\$$ returns relative to that of the $\sigma(R V)$-standardized returns.

\section{Standardization by GARCH(1,1) Volatility and by Forecasts of Realized Volatility}

Numerous parametric volatility models have been suggested in the literature for best capturing the conditional temporal dependencies in $\sigma_{t}$. The most commonly used specification is the simple univariate $\mathrm{GARCH}(1,1)$ model, and we follow standard practice by utilizing this as an 
illustrative benchmark for each of the two rates. That is, we posit that

$$
\sigma_{t}^{2}=\omega+\alpha r_{t-1}^{2}+\beta \sigma_{t-1}^{2}
$$

We refer to the associated estimates of the conditional standard deviations as $\sigma(G A R C H)$, with the $\sigma(G A R C H)$-standardized daily returns defined accordingly. ${ }^{6}$

Consistent with the prior literature, the summary statistics in the fourth panel of Table 1 show that standardization by $\sigma(G A R C H)$ reduces, but does not eliminate, the excess kurtosis. In particular, the sample kurtosis for the $\mathrm{DM} / \$$ drops from 5.4 to 4.8 , while the Yen $/ \$$ kurtosis is reduced from 7.4 to 5.4. Thus, in each case, significant excess kurtosis remains after the standardization.

It is natural to ask why such different results obtain for the $\sigma(R V)$ - versus the $\sigma(G A R C H)$-standardized returns. Of course, in general, we would expect different measures for $\sigma_{t}$ to affect the properties of the standardized returns. However, in this case, there is a specific aspect of the calculations that makes an obvious difference: $\sigma_{t}(R V)$ is an estimate of the volatility for the day-t returns conditional on the continuous (or high-frequency discrete intraday) sample path of stochastic volatility up to and including day t, whereas $\sigma_{t}(G A R C H)$ is an estimate of the

\footnotetext{
${ }^{6}$ For simplicity we focus on the univariate version of the various models along with the corresponding $\sigma$-standardized returns, but the same ideas carry over straightforwardly to more complicated multivariate volatility models. Furthermore, it is evident that an extensive specification search would provide alternative models with, at least, marginally improved predictive performance, but the qualitative results emphasized below are generic to the entire class of $\mathrm{ARCH}$ and stochastic volatility models.
} 
volatility of day-t returns conditional on the discrete sample path of returns up to but not

including day t.

To further underscore the importance of this difference, we next calculate $\sigma_{t}(R V F)$ as a one-day-ahead forecast of the realized volatility made at day $\mathrm{t}-1$, where the forecast formula is obtained by a projection of the realized volatility on the past daily realized volatilities. ${ }^{7}$ This approach is much closer in spirit to the $\sigma_{t}(G A R C H)$ estimator analyzed above, and we therefore conjecture that standardization by $\sigma_{t}(R V F)$ will reduce, but not eliminate, the excess kurtosis. ${ }^{8}$

For ease of comparison to the $\operatorname{GARCH}(1,1)$ case, we shall rely on a simple $\operatorname{ARMA}(1,1)$ structure for modeling the realized volatilities. Also, in direct analogy to the $\operatorname{GARCH}(1,1)$ case, the model is estimated over the full ten-year sample. ${ }^{9}$ From these estimates, we proceed with the creation of standard 1-day-ahead forecasts, from which we obtain our $\sigma_{t}(R V F)$ series, and

\footnotetext{
${ }^{7}$ The $\sigma(R V)$ forecasting exercise reported here is highly preliminary and stylized. A much more detailed analysis is currently being undertaken in Andersen, Bollerslev, Diebold and Labys (1999c). A related approach was recently pursued by Taylor and Xu (1997) in analyzing the informational content in high-frequency foreign exchange rates and volatilities implied in option prices.

${ }^{8}$ It is tempting to conjecture that $\sigma(R V F)$-standardized returns will be less fat-tailed than $\sigma(G A R C H)$ standardized returns, because $\sigma_{t-1}(R V)$ should provide a superior measure of recent volatility relative to $\sigma_{t-1}(G A R C H)$, which is operative in the $\operatorname{GARCH}(1,1)$ recursion. However, there is generally no simple relation between forecasts based on more relative to less information and the resulting amount of excess kurtosis of the corresponding standardized returns, as explained for example in Nelson (1996).

${ }^{9}$ Assuming no structural breaks during our 10-year sample, and that the dynamics remained unchanged relative to the previous ten years, we can justify the use of one-day-ahead volatility forecasts based on full-sample parameter estimates. Estimation with the full sample also has the obvious advantage that it avoids the early-on instability associated with recursive estimation.
} 
corresponding $\sigma(R V F)$-standardized returns.

The diagnostic statistics in the last panel of Table 1 show that the distributions of the $\sigma(R V F)$-standardized returns and the $\sigma(G A R C H)$-standardized returns are fairly similar. In particular, both exhibit fat tails relative to the normal. Figure 4 clearly reveals the reason behind this divergence between the $\sigma(R V)$-standardized returns, which to a first approximation appear Gaussian, and the leptokurtic $\sigma(R V F)$ - and $\sigma(G A R C H)$-standardized returns. The $\sigma_{t}(R V F)$ and $\sigma_{t}(G A R C H)$ volatility series are both one-day-ahead forecasts, and so are smoother than the object being forecast, which is effectively the $\sigma_{t}(R V)$ volatility series. Hence, standardization by $\sigma_{t}(R V F)$ or $\sigma_{t}(G A R C H)$ is insufficient to eliminate the excess kurtosis, whereas standardization by the $\sigma_{t}(R V)$ is able to accomplish that goal.

\section{Concluding Remarks and Directions for Future Research}

The normality of the $\sigma(R V)$-standardized returns is of special interest because it sheds light on the adequacy (or lack thereof) of commonly used continuous-time models of asset prices. Specifically, assume that the $\log$ price, $p_{t}$, follows a standard continuous time stochastic volatility diffusion, ${ }^{10}$

$$
d p_{t}=\sigma_{t} d W_{t}
$$

${ }^{10}$ The presence of a drift term in the diffusion does not affect any of the arguments given below, as long as the drift is independent of the volatility path over day $t$. 
where $t \geq 0, W_{t}$ is a standard Brownian motion, and $\sigma_{t}$ is a strictly stationary positive stochastic process. If $\sigma_{t}$ and $W_{t}$ are independent, it follows that the variance of the 1-day return, conditional on the sample path realization of the stochastic volatility process, is Gaussian with variance equal to the integrated volatility, $\int_{0}^{1} \sigma_{t-1+\tau}^{2} d \tau$. Hence, the integrated-volatility-standardized daily returns are standard normal,

$$
\frac{r_{t}}{\int_{0}^{1} \sigma_{t-1+\tau}^{2} d \tau} \sim N(0,1)
$$

But, as shown by Andersen and Bollerslev (1998) and Andersen, Bollerslev, Diebold and Labys (1999a), $\sigma_{t}(R V)$ is a highly efficient estimator of $\int_{0}^{1} \sigma_{t-1+\tau}^{2} d \tau$. Hence under the assumed conditions our $\sigma(R V)$-standardized returns should be approximately $\mathrm{N}(0,1)$, which we showed to be the case. $^{11}$

Our findings may be interpreted as providing indirect support for the assertion of a jumpless diffusion, because the presence of jumps is likely to result in a violation of the normality of the $\sigma(R V)$-standardized returns. Such inference would be consistent with the recent evidence in Das and Sundaram (1999), who arrive at this conclusion from a very different perspective, but counter to the findings in Andersen, Benzoni and Lund (1998), Bates (1996), Drost, Nijman and Werker (1998), and Johannes, Kumar and Polson (1998), among others, who argue for the

\footnotetext{
${ }^{11}$ It is noteworthy that these theoretical predictions hold up very well in practice in spite of the host of market microstructure features, such as non-synchronous trading and bid-ask bounce effects, which clearly invalidate the continuous-time diffusion assumption for the actual observed price process at the highest intraday sampling frequencies.
} 
importance of allowing for jumps in other markets and/or time periods. It is obviously of interest to pursue this issue through more formal and direct tests. The present study is relatively limited in scope, examining only two exchange rates, while much of the existing literature focuses on equities. Thus, our findings along this dimension are best interpreted as suggestive, and further work is required for a more extensive empirical assessment and reconciliation. Nonetheless, the documented ability to measure realized volatilities with good precision has opened an entirely new avenue for this line of inquiry. 


\section{References}

Andersen, T.G., Benzoni, L. and Lund, J. (1998), "Estimating Jump-Diffusions for Equity Returns,” Manuscript, Department of Finance, J.L. Kellogg Graduate School of Management, Northwestern University.

Andersen, T.G. and Bollerslev, T. (1998), “Answering the Skeptics: Yes, Standard Volatility Models Do Provide Accurate Forecasts," International Economic Review, 39, 885-905.

Andersen, T.G., Bollerslev, T., Diebold, F.X. and Labys, P. (1999a), "The Distribution of Exchange Rate Volatility," Wharton Financial Institutions Center Working Paper 99-08 and NBER Working Paper 6961.

Andersen, T.G., Bollerslev, T., Diebold, F.X. and Labys, P. (1999b), "Microstructure Bias and Volatility Signatures," Manuscript in progress.

Andersen, T.G., Bollerslev, T., Diebold, F.X. and Labys, P. (1999c), "Forecasting Realized Volatility: A VAR for VaR," Manuscript in progress.

Barndorff-Nielsen, O.E. and Shephard, N. (1998), "Aggregation and Model Construction for Volatility Models," Manuscript, Department of Mathematical Sciences, University of Aarhus, and Nuffield College, Oxford.

Bates, D.S. (1996), “Jumps and Stochastic Volatility: Exchange Rate Processes Implicit in Deutsche Mark Options," Review of Financial Studies, 9, 69-107.

Bollen, B. and Inder, B. (1999), "Ex Post, Unconditional Estimators of Daily Volatility," Manuscript, Department of Econometrics, Monash University.

Bollerslev, T. (1987), “A Conditional Heteroskedastic Time Series Model for Speculative Prices and Rates of Return," Review of Economics and Statistics, 69, 542-547.

Bollerslev, T., Chou, R.Y. and Kroner, K.F. (1992), “ARCH Modeling in Finance: A Selective Review of the Theory and Empirical Evidence," Journal of Econometrics, 52, 5-59.

Clark, P. K. (1973), “A Subordinated Stochastic Process Model with Finite Variance for Speculative Prices," Econometrica, 41, 135-155.

Das, S.R. and Sundaram, R.K. (1999), "Of Smiles and Smirks: A Term Structure Perspective," Manuscript, Graduate School of Business, Harvard University, and Stern School of Business, New York University.

Diebold, F.X., Gunther, T. and Tay, A.S. (1998), “Evaluating Density Forecasts, With 
Applications to Financial Risk Management," International Economic Review, 39, 863883.

Diebold, F.X., Hahn, J. and Tay, A.S. (1999), "Multivariate Density Forecast Evaluation and Calibration in Financial Risk Management: High-Frequency Returns on Foreign Exchange," Review of Economics and Statistics, 81, forthcoming.

Drost, F.C., T.E. Nijman and Werker, B.J.M. (1998), "Estimation and Testing in Models Containing Both Jumps and Conditional Volatility," Journal of Business and Economic Statistics, 16, 237-243.

Engle, R.F. and González-Rivera, G. (1991), “Semiparametric ARCH Models,” Journal of Business and Economic Statistics, 9, 345-359.

Hansen, B.E. (1994), “Autoregressive Conditional Density Estimation,” International Economic Review, 35, 705-730.

Hsieh, D.A. (1989), “Modeling Heteroskedasticity in Daily Foreign Exchange Rates,” Journal of Business and Economic Statistics, 7, 307-318.

Hsieh, D.A. (1991), “Chaos and Nonlinear Dynamics: Application to Financial Markets," Journal of Finance, 46, 1839-1877.

Johannes, M., Kumar, R. and Polson, N.G. (1999), "Jump Diffusion Models: Extraction of Jumps from Equity Indices,” Manuscript, Graduate School of Business, University of Chicago.

Nelson, D.B. (1996), "A Note on the Normalized Errors in ARCH and Stochastic Volatility Models," Econometric Theory, 12, 113-128.

Schwert, G.W. (1990) “Stock Market Volatility,” Financial Analysts Journal, May-June, 23-34.

Tauchen, G.E. and Pitts, M. (1983), “The Price Variability-Volume Relationship on Speculative Markets," Econometrica, 51, 485-505.

Taylor, S.J. (1986). Modelling Financial Time Series. Chichester, England: John Wiley \& Sons, Ltd.

Taylor, S.J. and Xu, X. (1997), "The Incremental Volatility Information in One Million Foreign Exchange Quotations," Journal of Empirical Finance, 4, 317-340. 
Table 1

Descriptive Statistics for Daily Exchange Rate Returns

\begin{tabular}{|c|c|c|c|c|c|c|c|c|c|c|}
\hline & \multicolumn{2}{|c|}{$\begin{array}{l}\text { Un- } \\
\text { Standardized }\end{array}$} & \multicolumn{2}{|c|}{$\begin{array}{l}\sigma(R V)- \\
\text { Standardized }\end{array}$} & \multicolumn{2}{|c|}{$\begin{array}{l}P(R V)- \\
\text { Standardized }\end{array}$} & \multicolumn{2}{|c|}{$\begin{array}{l}\sigma(G A R C H)- \\
\text { Standardized }\end{array}$} & \multicolumn{2}{|c|}{$\begin{array}{l}\sigma(R V F)- \\
\text { Standardized }\end{array}$} \\
\hline & $\mathrm{DM} / \$$ & Yen/\$ & $\mathrm{DM} / \$$ & Yen/\$ & $\mathrm{DM} / \$$ & Yen/\$ & $\mathrm{DM} / \$$ & Yen/\$ & $\mathrm{DM} / \$$ & Yen/\$ \\
\hline Mean & -0.007 & -0.009 & -0.007 & 0.007 & -0.007 & 0.016 & -0.002 & -0.011 & -0.001 & -0.013 \\
\hline Median & -0.010 & 0.007 & -0.017 & 0.015 & -0.017 & 0.028 & -0.003 & 0.017 & -0.016 & 0.011 \\
\hline Maximum & 3.909 & 5.445 & 3.040 & 2.971 & 3.040 & 2.557 & 5.370 & 5.954 & 5.782 & 7.400 \\
\hline Minimum & -3.333 & -3.682 & -2.753 & -2.747 & -2.753 & -2.704 & -4.814 & -4.681 & -4.470 & -5.261 \\
\hline Std. Dev. & 0.710 & 0.705 & 1.009 & 0.984 & 1.009 & 0.883 & 1.001 & 1.000 & 1.047 & 1.035 \\
\hline Skewness & 0.033 & 0.052 & 0.015 & 0.002 & 0.015 & -0.073 & -0.027 & -0.139 & 0.001 & -0.008 \\
\hline Kurtosis & 5.395 & 7.357 & 2.406 & 2.414 & 2.406 & 2.622 & 4.753 & 5.405 & 4.779 & 6.161 \\
\hline Correlation & 0.659 & & 0.661 & & 0.081 & & .661 & & 0.676 & \\
\hline
\end{tabular}


Figure 1

Quantile-Quantile Plots

Daily Exchange Rate Returns
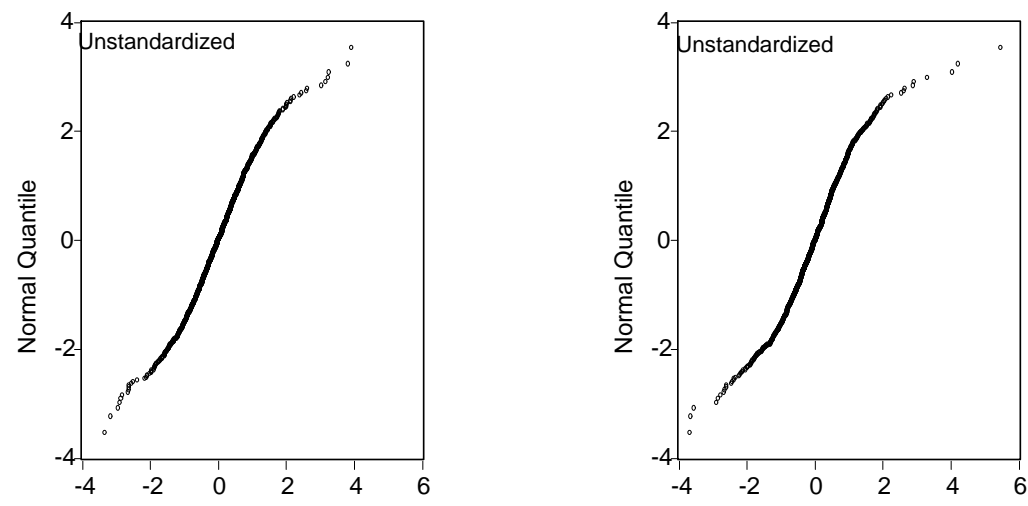

DM/\$ Return Quantile

Yen/\$ Return Quantile
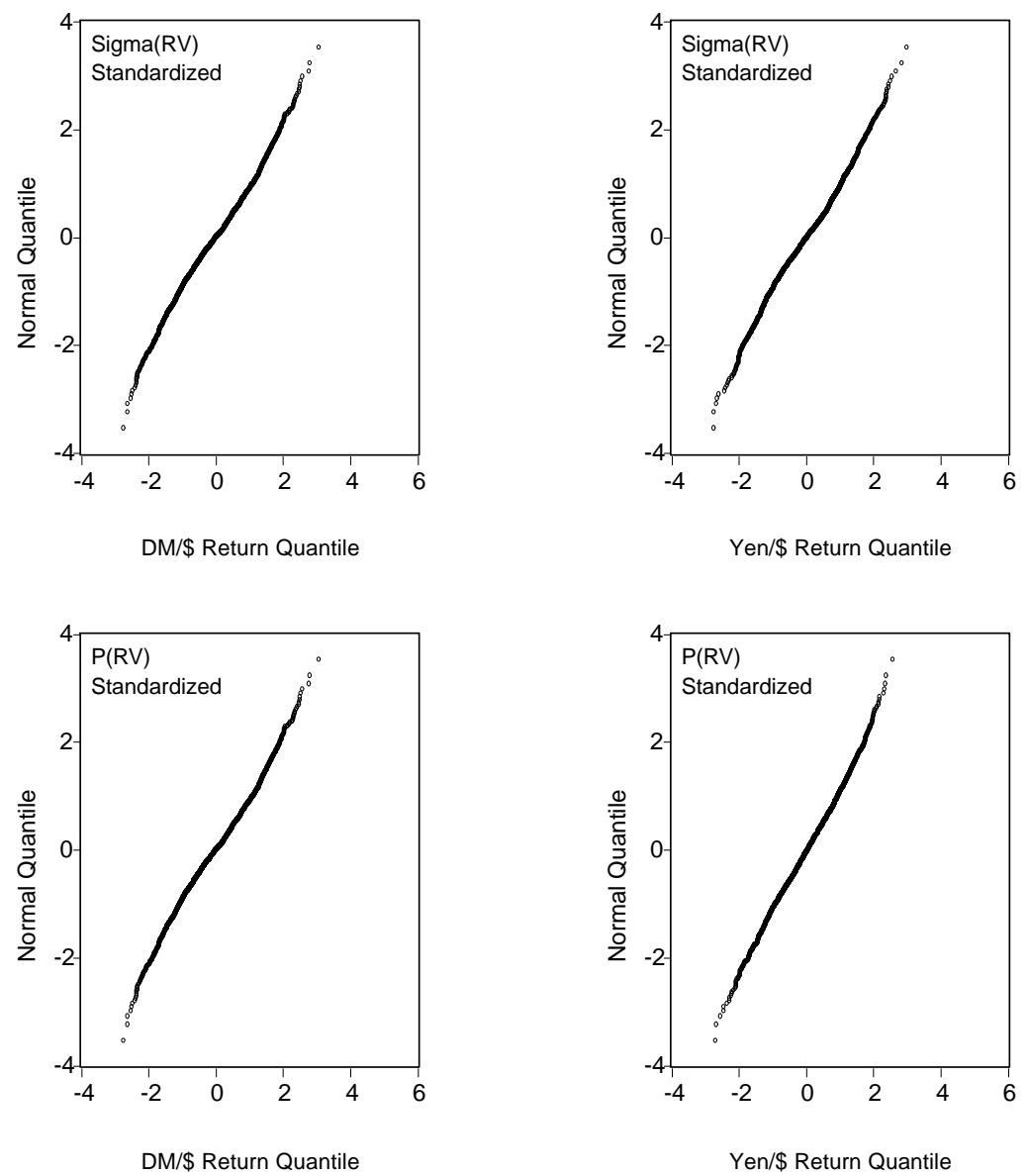

$-18-$ 


\section{Figure 2}

Scatterplots

\section{Daily Exchange Rate Returns}
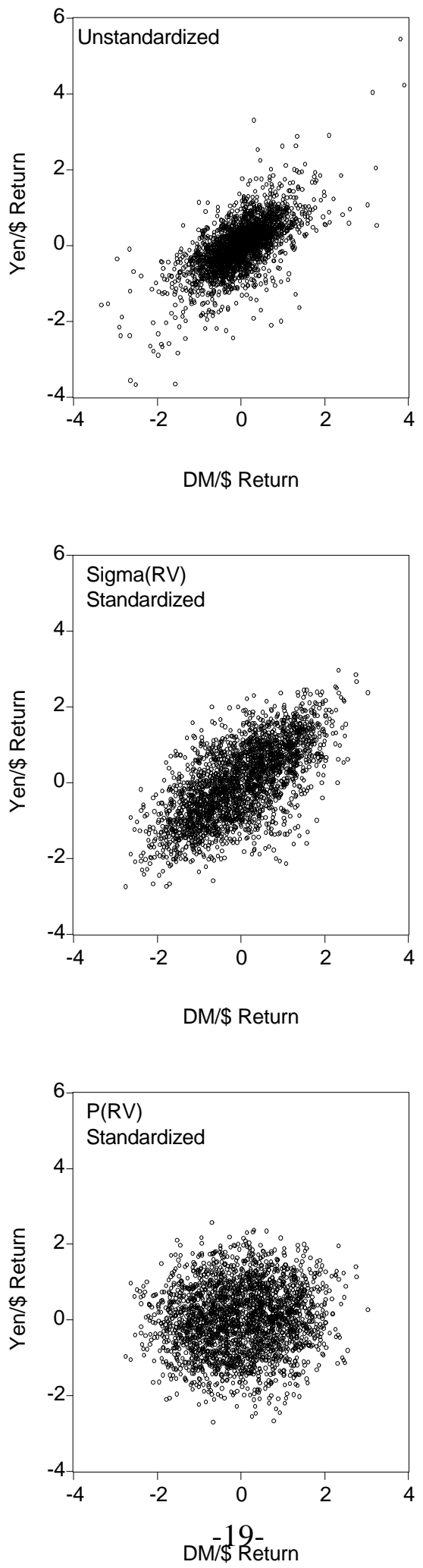
Figure 3

Sample Autocorrelation Functions

Daily Exchange Rate Returns
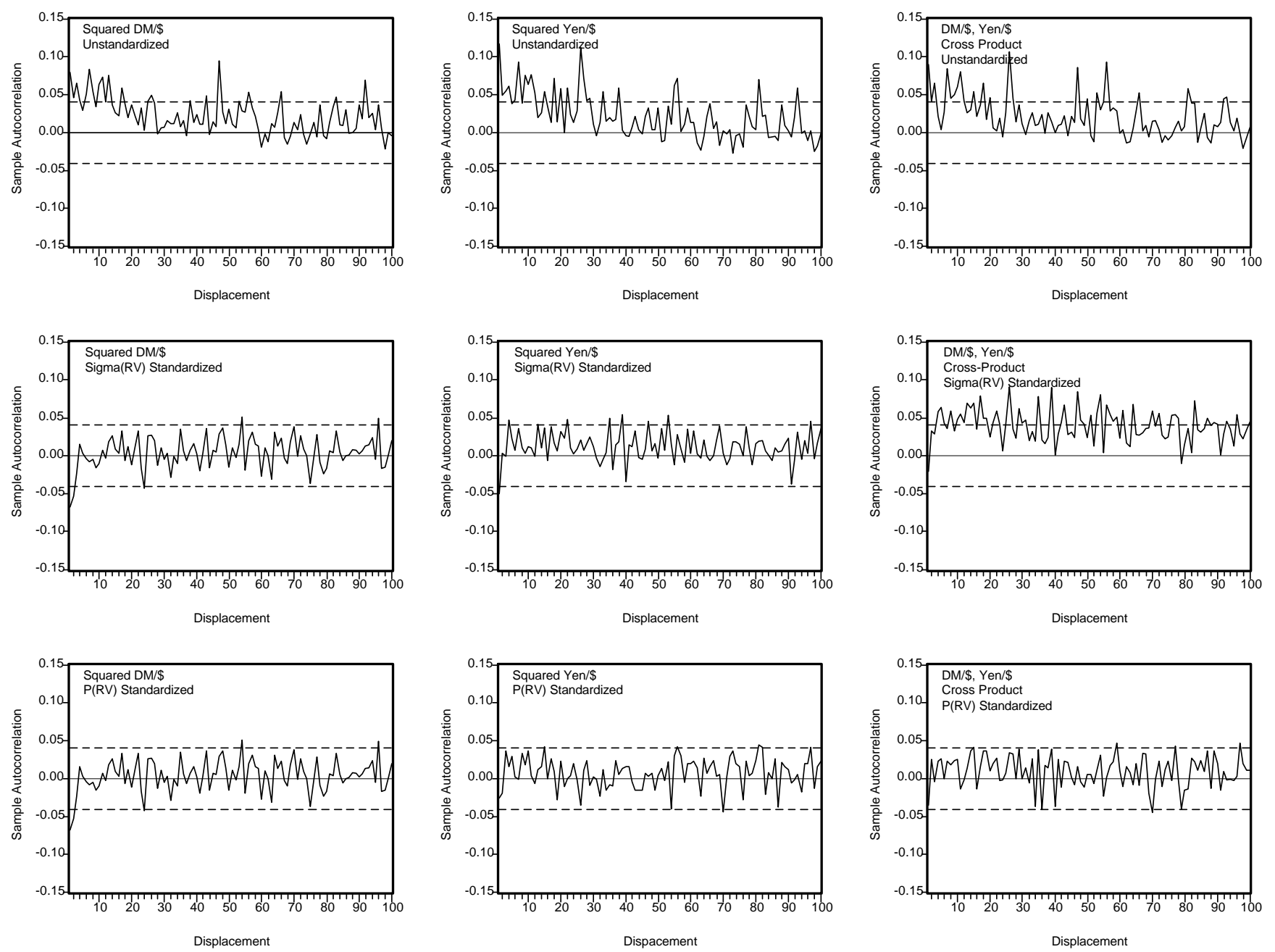
Figure 4

Time Series of Alternative Volatility Measures

Daily Exchange Rate Returns
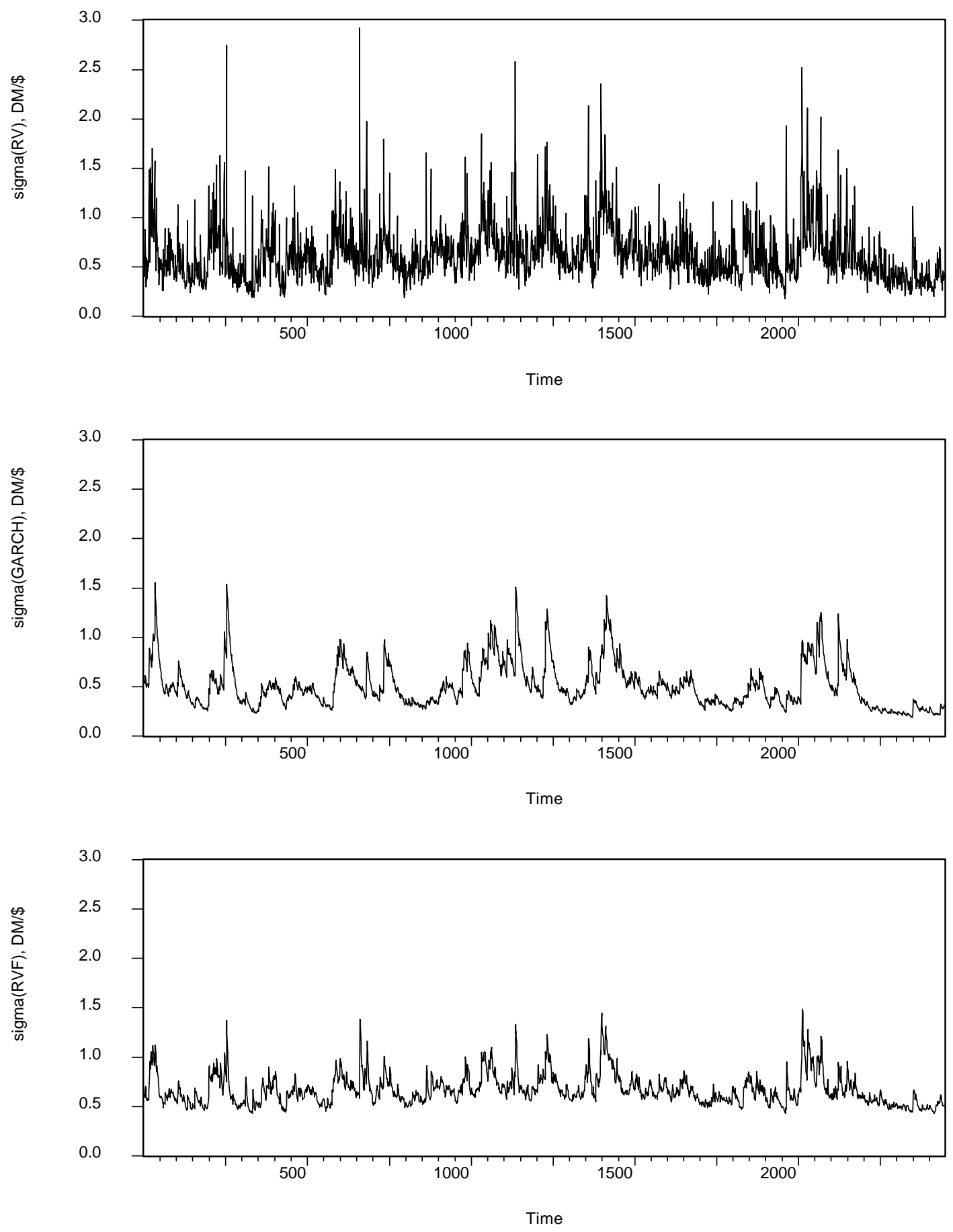